\section{$12+1$. SENTIMIENTOS DEL ALUMNADO UNIVERSITARIO DE EDUCACIÓN FÍSICA FRENTE A UNA PROPUESTA DE GAMIFICACIÓN: "GAME OF THRONES: LA IRA DE LOS DRAGONES”}

\author{
12 +1. FEELINGS OF PHYSICAL EDUCATION COLLEGE STUDENTS TOWARDS \\ A GAMIFICATION PROPOSAL: "GAME OF THRONES: THE ANGER OF THE \\ DRAGONS"
}

\author{
12 +1. SENTIMENTOS DO ACADÊMICO DE EDUCAÇÃO FÍSICA FRENTE A UMA \\ PROPOSTA DE GAMIFICAÇÃO: "GAME OF THRONES: A IRA DOS DRAGÕES"
}

Isaac Pérez López*, Enrique Rivera García*, Carmen Trigueros Cervantes*
Resumen: La propuesta de gamificación realizada tiene por objetivo evaluar la evolución de los sentimientos del alumnado a lo largo del proceso de aprendizaje; realizada en el contexto del aula universitaria de futuros docentes de Educación Física, han participado 50 estudiantes. Basada en la serie Juego de Tronos, metodológicamente se apoya en un análisis interpretativo de los diarios y reflexiones finales del alumnado. Los principales resultados los podemos centrar en: diferencia de sentimientos en función del sexo, que a lo largo del proceso se mantienen estables, dominando la idea de un aprendizaje desde el disfrute. En la focalización de los sentimientos, el docente mantiene una línea estable de transmisión de confianza, mientras que, frente a los compañeros de equipo y amigos, estos se muestran tanto en positivo como en negativo. Como conclusión, podemos decir que la gamificación como estrategia metodológica mejora la motivación del alumnado, logrando una mayor implicación en el aprendizaje.

Palavras chave: Educação superior. Pesquisa qualitativa. Capacitação de professores.

Resumo: A proposta de gamificação apresentada objetiva avaliar a evolução dos sentimentos de discentes no decorrer do processo de aprendizagem. 0 estudo foi realizado com 50 estudantes de Educação Física, tendo como contexto a aula universitária. Baseado na série Game of Thrones, o alicerce metodológico apoiou-se em análise interpretativa dos diários e reflexões finais dos atores investigados. Os principais resultados refletem diferenças de sentimentos em relação ao sexo, os quais permanecem inalterados ao longo do processo de aprendizagem, suscitando uma ideia de estudo como prazer. Os achados explicitam ainda que, concernente aos sentimentos, o docente mantém uma linha estável de transmissão de confiança, enquanto que em relação aos companheiros de equipe e amigos, demonstram sentimentos tanto positivos quanto negativos. Como conclusão, pode-se inferir que a gamificação, como estratégia metodológica, favorece a motivação do discente, revertendo-se em melhoria do processo de aprendizagem.

Abstract: The gamification proposal is aimed at evaluating the changes in students' feelings throughout the learning process. The proposal was carried out in the context of the university class of future Physical Education teachers, and it included 50 students. The proposal was based on the series Game of Thrones and relies methodologically on the interpretative analysis of the journals and on students' final reflections. The main results include: the difference of feelings according to sex, which remained stable during the process as the idea of learning from enjoyment prevailed. Regarding the focus of the feelings, the teacher maintains a stable line that conveys confidence, while feelings are both positive and negative regarding teammates and friends. In conclusion, that gamification as a methodological strategy improves student's motivation, which leads to greater involvement in learning.
*Universidad de Granada. Granada, España.

E-mail: isaacj@ugr.es;

erivera@ugr.es; ctriguer@ugr.es

Recebido em: 09-11-2018 Aprovado em: 02-05-2019 Publicado em: 11-06-2019

DOI: https://doi.org/10.22456/1982-8918.88031

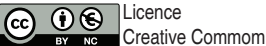




\section{INTRODUCCIÓN}

Las propuestas de innovación de la docencia universitaria, promovidas desde el Espacio Europeo de Educación Superior, radica en la posibilidad de contribuir a mejorar cualitativamente la práctica de la enseñanza y, en consecuencia, el proceso y los resultados de aprendizaje de los alumnos (MAURI; COLL; ONRUBIA, 2007). Este intento de cambio ha tenido lugar a diferentes niveles del proceso: planificación, metodología de aula, evaluación, organización de grupos, tiempo y espacio, etc. La mayoría de ellos se han quedado en cambios técnicos, olvidando en muchos casos que uno de los problemas a abordar es la falta de motivación del alumnado hacia el aprendizaje.

Una de las variables que más influye en el aprendizaje es el clima de aula (PIANTA et al., 2007). Junto a los aspectos relacionados con la metodología desarrollada en clase, el alumnado universitario valora especialmente la capacidad del profesorado para crear un buen clima emocional (GONZÁLEZ RODRÍGUEZ et al., 2010). Para Casassus (2007), la diferencia de logros en el aprendizaje del alumnado, más que en la técnica utilizada, se encuentra principalmente en los climas emocionales del aula.

El aprendizaje se favorece en un entorno en el que se puedan establecer relaciones interpersonales positivas, donde el alumnado se sienta apreciado, reconocido, respetado y validado (MCCOMBS; WHISLER, 2000). Y es que, como se desprende del trabajo de Grieve (2010), uno de los principales indicadores de excelencia en la docencia se encuentra en el desarrollo de habilidades interpersonales en el aula entre el profesorado y los estudiantes.

Por tanto, el clima de clase dependerá en gran medida del profesor (HERNÁNDEZ ÁLVAREZ, 2000), él será el principal responsable de que el alumnado adopte una predisposición positiva hacia el aprendizaje, aumentando su motivación. Como expresa Estebaranz (2001), uno de los medios para su logro debe ser transfiriéndole el protagonismo de su proceso formativo, ofreciéndole propuestas que le sean significativas y atractivas y cuestionando lo que ya saben.

Necesitamos salir de la exposición magistral. No se trata de eliminarla por completo del proceso, pero sí sustituir su protagonismo y por ende el del docente, propiciando la participación activa del alumnado en el aprendizaje. Queremos un alumnado que se admire ante el conocimiento, que sienta afecto y gratitud en sus relaciones, que vea de forma optimista el proceso de aprendizaje. Queremos que sus sentimientos hacia nuestras clases no estén presididos por el enfado, la tristeza, la indignación que provoca el sentirse injustamente tratado y por qué no decirlo el odio hacia todo lo que signifique nuestra materia; pero sin perder de vista que su presencia también puede ser abordada en positivo. Pasamos de la emoción al sentimiento, pero teniendo siempre presente que ambos conceptos son raramente inseparables, que la barrera que los separa se sitúa en la evaluación consciente, previa a la manifestación de un sentimiento (DAMASIO, 2005).

Nuestra herramienta para provocar sentimientos y emociones positivas hacia el aprendizaje ha sido la gamificación. Entre las definiciones más extendidas de este nuevo concepto destaca la Deterding y colaboradores (2011), entendiéndola como el uso de elementos de diseño del juego en contextos de no juego. Kapp (2012) señala que se trata de utilizar las mecánicas del juego, su estética y sus estrategias para involucrar a la gente, motivar la acción, promover el aprendizaje y resolver problemas. Y todo ello con el objetivo de 
modificar o promover comportamientos deseados (HUOTARI; HAMARI, 2012; LEE; HAMMER, 2011).

Como concepto emergente se ha convertido en una tendencia social con un gran impacto a nivel mundial, fundamentalmente desde el segundo semestre de 2010 (GROH, 2012), y que en España comienza a extenderse en 2011 (GAME MARKETING, 2012). Su aplicación se ha desarrollado principalmente en el área del marketing, aunque también se pueden encontrar ejemplos relacionados con la salud, el medio ambiente, los medios de comunicación, el entretenimiento o la educación.

A pesar de que la educación es un área con gran potencial para la aplicación de estrategias de gamificación (DANOWSKA-FLORCZYK; MOSTOWSKI, 2012; ERENLI; 2012; SIMÕES, et al. 2012; SIMÕES, DÍAZ; FERNÁNDEZ, 2013), aún es necesario llevar a cabo un mayor número de investigaciones que permitan profundizar en las posibilidades que ofrecen en el ámbito educativo (DETERDING et al, 2011; MUNTEAN, 2011) y que, de este modo, se sumen a las ya existentes con alumnado universitario como es el caso, por ejemplo, de los trabajos de Carolei, (2012), Carpena, Cataldi y Muñiz (2012) o Kumar (2012).

Nuestra propuesta de gamificación "GamE oF trhones: la ira de los dragones", parte de la voluntariedad. Nuestro referente principal se situará en la motivación intrínseca (HUOTARI; HAMARI, 2012; MELCHOR, 2012). Para todos aquellos que desconocen esta serie televisiva pasamos a una breve exposición de las claves utilizadas.

Como en la serie original, en esta particular adaptación el alumnado se dividió en diferentes Casas: llíbers, Melgarian, Lothbrok, Kudlister y Lannsinef, cada una de ellas contaba con lema y estandarte. Cada una de con tres roles principales: Señor de la familia, Primogénito y Estirpe (resto de integrantes de la casa). En una trama paralela (pero con incidencia en la anterior) teníamos a la Guardia de la Noche (defendiendo "El Muro") y los Caminantes Blancos, incluyendo la figura del Lord Comandante.

Cada casa gobernaba un reino diferente, que se correspondía con uno de los bloques de contenidos del currículum de Educación Física:

Cuadro 1- Reinos de la propuesta de Gamificación.

\begin{tabular}{|c|c|c|c|c|}
\hline Bloque Curricular & $\begin{array}{c}\text { Condición Física- } \\
\text { Salud }\end{array}$ & Juegos y Deportes & Expresión Corporal & $\begin{array}{c}\text { Actividades en el } \\
\text { medio natural }\end{array}$ \\
\hline Reinos & Físicor & Deporticia & Expresanto & Naturalia \\
\hline
\end{tabular}

Fuente: elaboración propia

Además, estaban los habitantes de Nido del Rey (Desembarco del Rey en la serie), donde vivía el Rey de Poniente, la Mano del Rey, su Lord Comandante y el resto de la población. $Y$ todos ellos se ubican, por tanto, en una zona diferente dentro del mapa de Poniente entorno al que se configuró la propuesta (figura 1): la provincia de Granada. 
Figura 1 - Mapa de Poniente en "gamE oF thrones: la ira de los dragones"

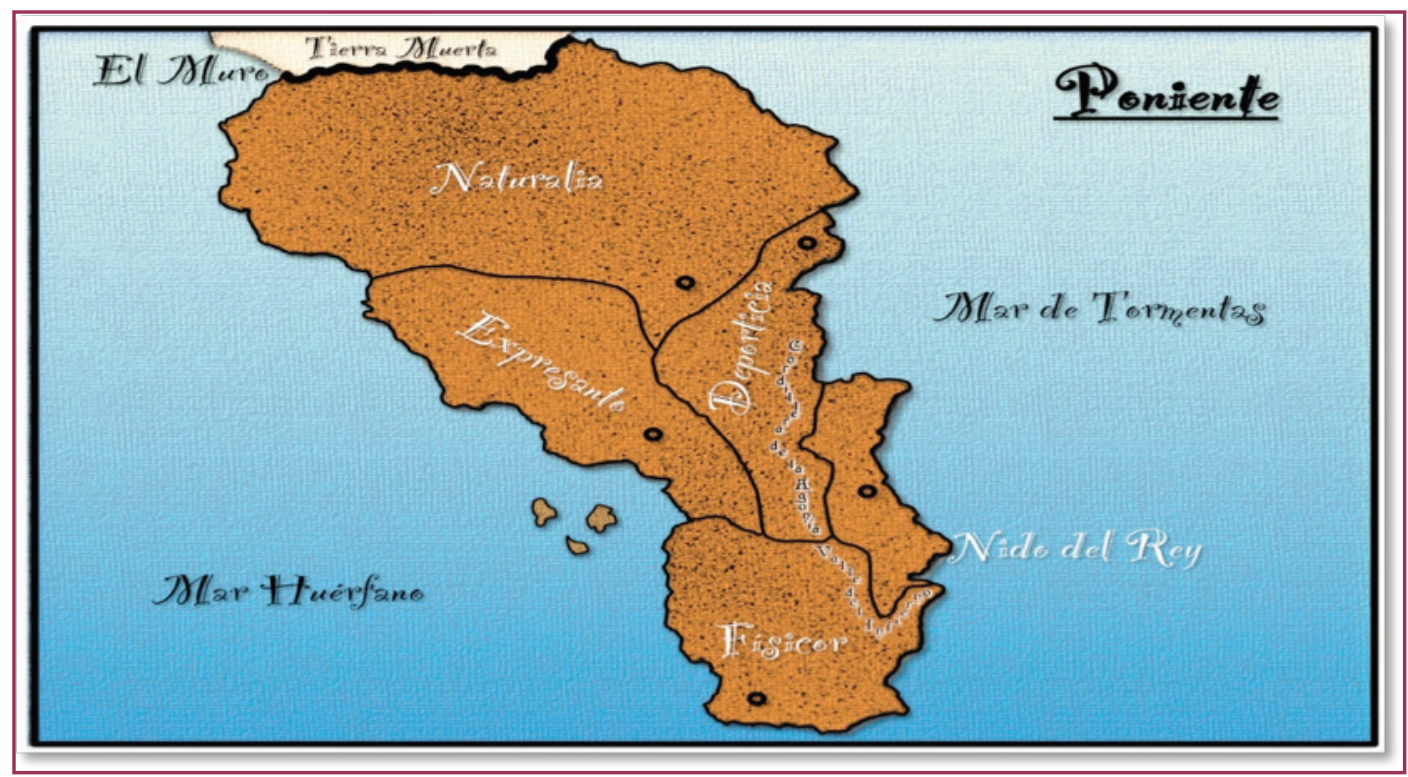

Fuente: elaboración propia

En función del rol que desempeña cada participante, inicia con una calificación diferente, que iba desde el 10 del Rey de Poniente hasta el 5 de los integrantes de la Guardia de la Noche. Los Señores de cada reino fueron quienes lograron realizar mejor el primer reto que se les planteó, consistente en lograr el mayor número de apoyos a su "candidatura". Posteriormente, los diferentes grupos se confeccionaron al azar. El rol de cada participante en su grupo se estableció de forma consensuada; en el segundo capítulo se decidió quién comenzaría siendo el Rey de Poniente. A lo largo de la aventura, dicho estatus se fue modificando en función de los logros alcanzados y de la resolución de los diferentes retos formativos planteados.

Esta circunstancia, además de mantener la coherencia existente con la serie original, se planteó intencionadamente con la finalidad de poder valorar las diferentes reacciones que, como era de esperar, tuvieron los alumnos ante la desigual situación de partida de cada uno de ellos y, posteriormente, reflexionar sobre cómo habían gestionado dicha situación.

El planteamiento desarrollado no consistió en la secuencia de una serie de clases presenciales magistrales, sino que los propios alumnos fueron quienes determinaron su propio ritmo de aprendizaje, en función de las actividades que el desarrollo de la propuesta les proponía. Se dividió en 10 capítulos (sesiones presenciales -tabla 1-), al igual que las temporadas de Juego de Tronos, donde tenían lugar diferentes acontecimientos relevantes, fundamentalmente a través de dinámicas de organización recreativas que combinaban aspectos teórico-prácticos, físicos-cognitivos y emocionales (con unos objetivos y contenidos concretos relacionados con la asignatura, pero dentro de la ambientación de la serie). 
Cuadro 2 - Título y sinopsis de los 10 capítulos que componen la primera temporada de "GamE oF thrones: la ira de los dragones"

\begin{tabular}{|c|c|c|}
\hline Capítulo & Título & Sinopsis \\
\hline 1 & "Paranoia” inicial & $\begin{array}{l}\text { Presentación de "gamE oF thrones: la ira de los dragones", en la que } \\
\text { todos los alumnos se comprometen a participar activamente en la } \\
\text { propuesta y a mejorar su formación. }\end{array}$ \\
\hline 2 & El origen de Poniente & $\begin{array}{l}\text { Mediante una intensa "batalla" se decide quién se sentará en el Trono } \\
\text { de Hierro y los reinos a ocupar por cada Casa. Además, el ganador del } \\
\text { enfrentamiento entre la Guardia de la Noche y los Caminantes Blancos } \\
\text { elige el Lord Comandante del ejército rival. }\end{array}$ \\
\hline 3 & $\begin{array}{c}\text { El origen de Poniente } \\
\text { II }\end{array}$ & $\begin{array}{l}\text { En la segunda parte de este capítulo cada Casa y Ejército se presenta } \\
\text { ante el resto, y el Árbol corazón (profesor), mediante una performance, } \\
\text { caracterizados según sus personajes en la aventura }\end{array}$ \\
\hline 4 & $\begin{array}{l}\text { Provisiones para el } \\
\text { Invierno }\end{array}$ & $\begin{array}{l}\text { Los habitantes de Poniente tienen que recopilar y presentar ante el Árbol } \\
\text { corazón el mayor número de evidencias de la imagen que la sociedad } \\
\text { actual tiene de la E.F., analizar los motivos que han dado lugar a ellas y } \\
\text { proponer propuestas de mejora. }\end{array}$ \\
\hline 5 & $\begin{array}{l}\text { La llegada del } \\
\text { Invierno }\end{array}$ & $\begin{array}{l}\text { Las diferentes Casas y Ejércitos deben demostrar quién es capaz de } \\
\text { afrontar mejor las "inclemencias del invierno", evidenciando su capacidad } \\
\text { de trabajo en equipo y su competencia a la hora de planificar. }\end{array}$ \\
\hline 6 & $\begin{array}{l}\text { Celebración de las } 7 \\
\text { semanas de reinado }\end{array}$ & $\begin{array}{l}\text { Tras el juramento de lealtad al Rey de Poniente por parte de los Señores } \\
\text { de cada reino, se celebran unos juegos en los que la recompensa para el } \\
\text { ganador será una importante suma de dinero para mejorar sus "armas" } \\
\text { para las "batallas". }\end{array}$ \\
\hline 7 & $\begin{array}{c}\text { Celebración de la } \\
\text { boda de los Reyes de } \\
\text { Poniente }\end{array}$ & $\begin{array}{l}\text { Se celebra la boda de los nuevos Reyes de Poniente, y tras ello, la Casa } \\
\text { que alcanza la victoria en los juegos establece parentesco con ellos } \\
\text { mediante el enlace de sus primogénitos. }\end{array}$ \\
\hline 8 & $\begin{array}{l}\text { Celebración del } \\
\text { derrocamiento } \\
\text { del primer Rey de } \\
\text { Poniente }\end{array}$ & $\begin{array}{l}\text { Con la intención de celebrar el derrocamiento del Rey Xela llíberis (primer } \\
\text { Rey de Poniente) se organizan unos juegos entre todas las Casas para } \\
\text { determinar el mejor guerrero de cada una de ellas y de todo Poniente. }\end{array}$ \\
\hline 9 & \#changEFiscoming & $\begin{array}{l}\text { Los habitantes de Poniente llevan a cabo un debate en twitter, iniciado } \\
\text { por la Mano del Rey, con la finalidad de concienciar sobre la necesidad } \\
\text { de un cambio en el planteamiento tradicional de la E.F. }\end{array}$ \\
\hline 10 & La ira de los dragones & $\begin{array}{l}\text { En este último capítulo, Daenerys Targaryen aparece con sus } 3 \text { dragones } \\
\text { para reivindicar que es la legítima heredera de las enseñanzas del Árbol } \\
\text { corazón, dando lugar a una apasionante voleytalla final. }\end{array}$ \\
\hline
\end{tabular}

Fuente: elaboración propia

En un intento de favorecer la credibilidad de lo que se estaba recreando, los integrantes de cada reino tenían tres actividades fundamentales a realizar:

a) Entrenar o Prepararse (para las "batallas"): Realizando diversos retos formativos donde los alumnos desarrollan los diferentes contenidos de la asignatura, y en los que se favorece fundamentalmente el desarrollo de la conciencia crítica y la creatividad.

b) Avanzar (a través de los reinos): En un intento de simular la progresión de los reinos hacia Nido del Rey para hacerse con el Trono de Hierro (que era su objetivo dentro de nuestra aventura/"serie"). Las distintas Casas debían realizar un avance "real". Como en la serie suele hacerse andando, a caballo o en barco, aquí se simbolizó corriendo, en bici o nadando. De este modo se fomentó la práctica de actividad física en el alumnado; 
cada integrante de una determinada "Casa" debía realizar esas actividades durante una semana, teniendo que cumplir una distancia y tiempos mínimos en cada jornada. Todo ello tenía que ser registrado individualmente mediante una aplicación móvil como, por ejemplo, Endomondo o Runtastic.

c) Luchar (contra otras Casas): Con la intención de ir acercándose hacia Nido del Rey. Para ello se organizaron actividades muy diversas que simulaban "batallas" entre las distintas Casas o Ejércitos, en algunos casos aprovechando localizaciones dentro de la ciudad de Granada (como parques o plazas) que ofrecen un entorno propicio para llevarlas a cabo. Y que, a pesar de ser espacios no convencionales en el ámbito universitario, son muy significativos para el alumnado e idóneos para generar gran cantidad de aprendizajes.

Paralelamente debían trabajar para dar con la fórmula que les permitiera forjar "acero valyrio" (que es la aleación de un material que da lugar a la fabricación de las mejores armas en Poniente), lo que representaron tratando de diseñar el mejor proyecto (o Unidad didáctica) que cada Casa o Ejército era capaz de construir (que es uno de los principales objetivos de la asignatura).

De cada reto formativo, así como de la evolución del acero valyrio que estuvieran forjando, fueron recibiendo continuamente feedback por parte del "Árbol corazón" (el profesor). Y, además, lograban diferentes beneficios o ventajas en función del resultado obtenido en dichas actividades que, posteriormente, podían usar en las "batallas" que llevaban a cabo.

\section{DISEÑO METODOLÓGICO}

La evaluación del proceso narrado se ha enmarcada en el paradigma interpretativo. Al querer conocer los sentimientos, creemos que la metodología cualitativa es la más adecuada, siguiendo las líneas trazadas para el diseño, análisis e interpretación marcadas por Denzin y Lincoln (2012).

\subsection{OBJETO Y OBJETIVOS DE LA INVESTIGACIÓN}

Evaluar la evolución de los sentimientos del alumnado universitario a lo largo del proceso de aprendizaje, utilizando una propuesta de gamificación como metodología de enseñanza. Como objetivos específicos nos proponemos indagar en los sentimientos que ha generado la propuesta al inicio, durante y al final del proceso; analizar las posibles diferencias por sexo y rol que se producen en la propuesta. Describir e interpretar la presencia o ausencia de sentimientos.

\subsection{CONTEXTUALIZACIÓN}

La experiencia se ha llevado a cabo con estudiantes del grado de Ciencias de la Actividad Física, dentro de la asignatura de Didáctica de la Educación Física desarrollada en el quinto semestre de carrera. Han participado en ella un total de 16 mujeres y 43 hombres, lo que hace un total de 59 participantes. 


\subsection{TÉCNICA DE PRODUCCIÓN DE LA INFORMACIÓN}

La producción de la información se ha realizado a través de una breve narrativa sobre la experiencia que los estudiantes (MÉNDEZ-LEITE; CORTÉS-GONZÁLEZ; RIVAS-FLORES, 2017; PÉREZ-SAMANIEGO; FUENTES; DEVÍS-DEVÍS, 2011). Para ganar en validez, se ha dejado esta opción como voluntaria y anónima, siendo realizada al finalizar cada uno de los capítulos (clases). La técnica de producción de la información se ha apoyado en un cuestionario abierto de Google Drive. Al disponer de la fecha de realización, esto nos ha permitido agrupar las aportaciones de los estudiantes en cuatro momentos: Presentación; Etapa Inicial; Intermedia y Final.

\subsection{PROCEDIMIENTO DE ANÁLISIS}

El proceso de análisis ha sido emergente, trazado desde la inductividad y respetando las teorías y creencias construidas por los participantes. Podemos enmarcar el modelo de análisis dentro de un enfoque categorial y metafórico, ya que ha tratado de hacer visibles las principales metáforas (sentimientos) construidas por los participantes, al tiempo que se ha realizado su categorización y codificación. Apoyándonos en el software NVivo 11 Plus, el punto de partida ha sido una frecuencia de palabras global, donde se han identificado los adjetivos en aquellas unidades de significado codificadas como sentimientos. De esta primera categorización y codificación, hemos seleccionado los 13 sentimientos $(12+1)$ con mayor presencia. En segundo nivel, se ha realizado una codificación en base a los actores a los que se hacía referencia: docente, compañeros y amigos. En un tercer nivel se ha cruzado la información mediante matrices de codificación, para localizar las relaciones en los planos anteriormente descritos: Similitudes y diferencias por sexo; evolución de los sentimientos en cada una de las fases del proceso y relaciones existentes entre las emociones expresadas y los actores presentes en la propuesta.

\section{ANALISIS E INTERPRETACIÓN DE RESULTADOS}

Los resultados y su interpretación parten del análisis de los discursos presentes en los estudiantes. Van a dar respuesta a tres interrogantes respectos a los sentimientos presentes: ¿Exiten diferencia entre los alumnos y las alumnas? ¿Cómo evolucionan a lo largo de cada una de las etapas? ¿Hacia que actores del proceso se dirigen? Esta va a ser nuestra columna vertebral, en torno a ella se irán conformando las diferentes teorías sustantivas fruto del cruce de las teorías de los participantes, de los investigadores y las formales ya construidas. Los trece sentimientos que construyen el análisis los podemos ver en el Cuadro 3:

Cuadro 3- Sentimientos con mayor presencia en las narrativas de los participantes

\begin{tabular}{|c|c|c|c|c|c|}
\hline \multicolumn{3}{|c|}{ Sentimientos positivos } & \multicolumn{3}{c|}{ Sentimientos negativos } \\
\hline Satisfacción & Disfrute & Sorpresa & Nerviosismo & Frustración & Incertidumbre \\
\hline Emoción & Ilusión & Confianza & Ansiedad & Estrés & Decepción \\
\hline Alegría & \multicolumn{3}{|l}{} \\
\hline
\end{tabular}


Por orden de presencia en las narrativas, la "satisfacción" es la que tiene mayor presencia, seguido del sentimiento de "disfrute", "sorpresa" y "emoción". Estos cuatro ocupan los primeros lugares, siendo la "ansiedad", "estrés" y "decepción" quienes ocupan los últimos puestos como sentimientos vividos a lo largo del proceso de aprendizaje.

\subsection{EMOCIÓN Y SEXO EDUCADO}

Son múltiples los estudios centrados en la neurociencia, (SPALEK et al., 2015) que vienen identificando que hay diferencia entre las emociones y sentimientos percibidos por los hombres y las mujeres ante diferentes situaciones. En el análisis de nuestros datos se confirma esta teoría desde un simple cruce de los sentimientos expresados por los hombres y las mujeres (Grafo 1):

Grafo 1 - Las emociones en función del sexo de los participantes

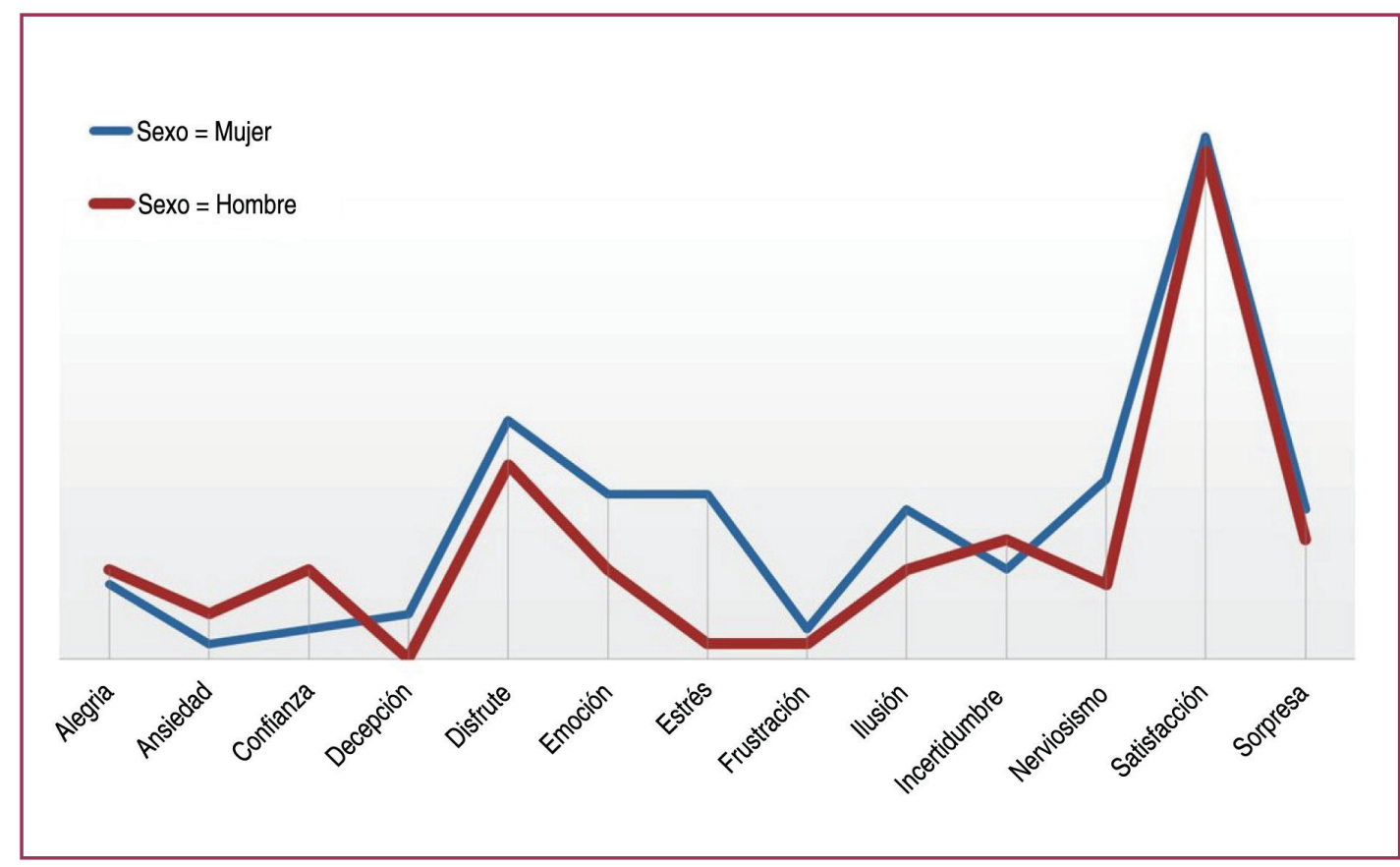

Fuente: elaboración propia

Las alumnas presentan menor ansiedad, confianza e incertidumbre en sus manifestaciones. Los alumnos, mayor presencia de disfrute, emoción, estrés, ilusión, nerviosismo y frustración. La ansiedad, confianza e incertidumbre, son emociones que claramente superan en presencia en los chicos, situándose el resto en un plano de igualdad o inferioridad. A vista de pájaro en las alumnas hay mayor presencia emocional que en los alumnos. Las diferencias fundamentales se sitúan en la confianza, el estrés y el nerviosismo.

Poner la confianza "en los demás miembros del equipo, sacar lo máximo de uno mismo, sentirte parte de un todo" ( +5 ); detrás de estas palabras se evidencia la consolidación del trabajo en equipo. Pero si en las alumnas es una emoción que se aprecia en positivo, en los alumnos se muestra con doble cara: la negativa, identificada como una pérdida de confianza en los amigos de siempre al tener que competir contra ellos. Pero este espíritu agonista es el que los lleva a buscar la confianza en sí mismo y en su equipo: 
Este día lo llevábamos esperando durante mucho tiempo y si soy sincero desde el primer día tenía plena confianza en que lo conseguiríamos! Delante teníamos un reto muy complicado, pero a nosotros como he dicho en muchas ocasiones nos gustan las adversidades y nos crecemos ( 99$)$.

La presencia de la competencia es algo a tener en cuenta; crítica habitual a la gamificación, pone en peligro el clima de aula ante la posible pérdida del objetivo principal por el ansia de lograr la victoria por encima de todo (WERBACH; HUNTER 2012).

En segundo lugar, apreciamos diferencias respecto a la ilusión, inferior en las chicas: "Es necesario plantear cosas nuevas, salir de esa "zona de confort" e introducirse nuevos retos que ilusionen a los que están a tu alrededor" ( por cambiar la formación inicial y basarla en la participación del alumnado en la construcción de sus aprendizajes.

Las alumnas, vuelcan gran parte de sus ilusiones en lograr la aprobación del "árbol corazón" (el docente) y admiran su dedicación y trabajo al proyecto, es la "ilusión [de] recibir ese mensaje del árbol corazón dándome ánimos en el que la posdata me hizo reflexionar mucho (siempre me pasa eso con sus cuervos) (ô 24).

Por último, el estrés se manifiesta de forma contundente en las chicas. ¿Hacia dónde lo enfocan? Hacia la resolución de los retos que plantea el docente. Lo ven complejo de gestionar, pero conscientes de su control:

[...] se acerca el invierno y queremos tener el mayor número de provisiones y de la mayor calidad posible. La verdad es que estamos estresados, para qué nos vamos a engañar, que si foto por aquí, grabación de voz por otro lado, vídeos en diferentes escenarios [...] (क 16).

Otra manifestación del estrés la tenemos en la competición. El agonismo predomina en el proceso y lo mediatiza. Esta situación nos lleva a pensar a limitar los enfrentamientos directos que erosionan la autoestima y ponen en riesgo el clima de aula. Estamos formando docentes para educar en ciudadanía, no ejecutivos de Wall Street.

Estrés que se puede manifestar también en la falta de recursos para ejercer el liderazgo en el grupo, tal y como expresaba una alumna al comentar que estaba "cabreada [consigo] conmigo misma por no haber sido capaz de llegar a un consenso sobre la situación que más nos convenía a todos". ( 9 12).

\subsection{EL CARRUSEL DE LOS SENTIMIENTOS Y LA CONSTRUCCIÓN DEL CLIMA DE AULA}

En una primera aproximación (Grafo 2), la presentación se caracteriza por: el sentimiento de satisfacción y la incertidumbre ante la posibilidad de disfrutar en la clase. En la fase Inicial, repite los sentimientos anteriores, sumando a ellos el estrés. En la Intermedia se mantienen las presentes, pero entra la incertidumbre en clave positiva. Por último, en la fase Final se estabilizan los sentimientos decreciendo los comentarios en torno a la satisfacción. 
Grafo 2 - Evolución de los sentimientos a lo largo de las etapas de la propuesta

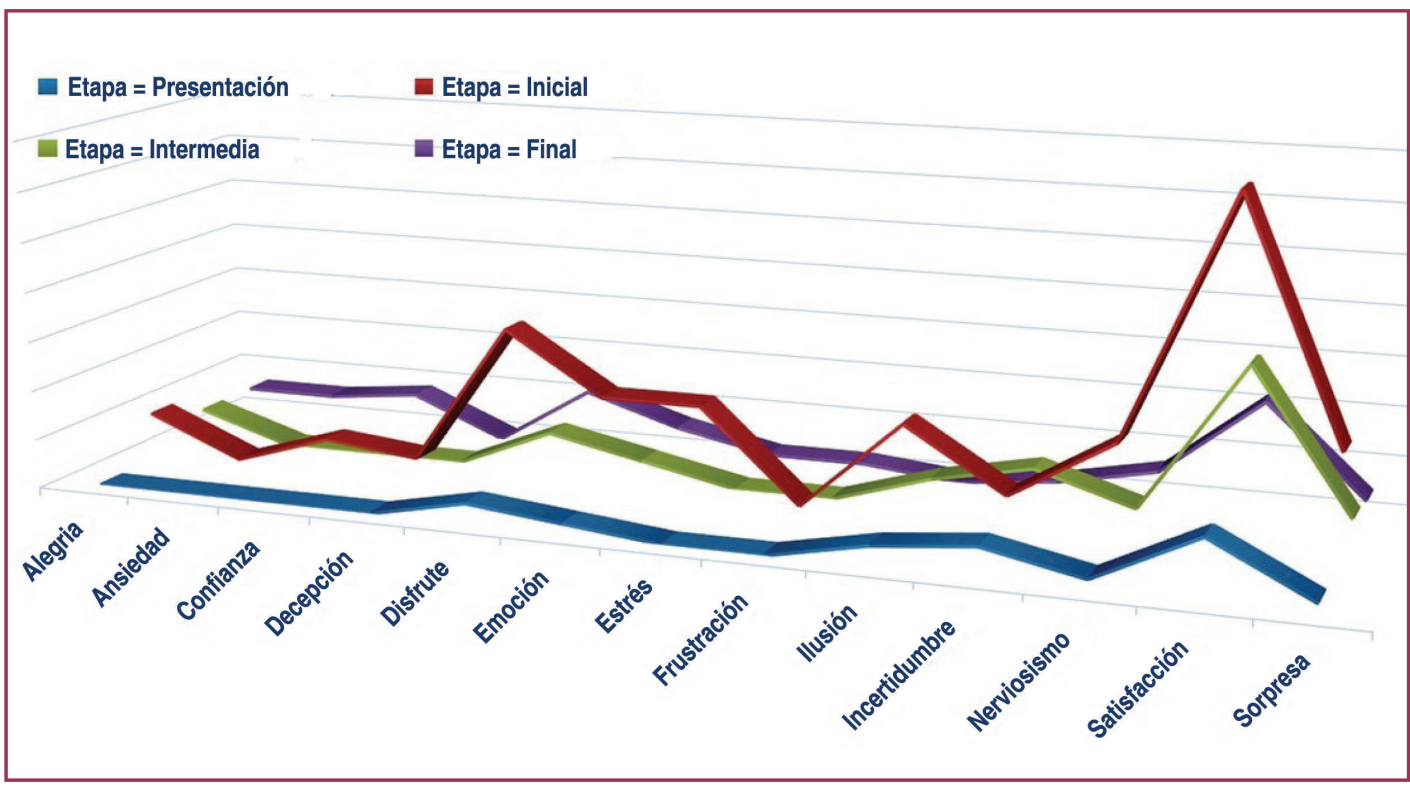

Fuente: Elaboración propia

La satisfacción es un sentimiento que se ha mantenido constantes a lo largo de todo el proceso. Nuestra pregunta en este caso tiene que enfocarse hacia dos direcciones: con qué 0 quienes se han sentido satisfechos, y por qué. estos van a ser los dos ejes de nuestro análisis.

En esta cita podemos encontrar las primeras respuestas:

Me ha parecido muy creativa, .... me ha impresionado un montón ..... me encanta simular juego de tronos y me ha sorprendido el nivel de detalles, ... se nota que hay un gran trabajo detrás de ésta y, así como a los profesores les gusta ver que sus alumnos se implican en la asignatura yo también valoro mucho el esfuerzo. ( $q$ 15).

Siguiendo a Herranz (2013), hay que impresionar, desde la diferencia, crear propuestas novedosas bien preparadas desde el inicio, evidenciar que la exigencia al alumnado viene precedida por la exigencia propia a nosotros mismos como docentes. Hay que provocar que se generen sentimientos y emociones de satisfacción en el alumnado (BEZA, 2011). Romper con la rutina, evitar que los estudiantes te etiqueten dentro del grupo de lo normalizado. Como manifiesta un alumno, si preguntas a compañeros de cursos superiores "te pueden describir exactamente lo que se hace en cada asignatura sin equivocarse ni un ápice, sin dar lugar a la incertidumbre 0 novedad". (ठ 17).

La confianza en sus iguales que manifiesta una alumna nos llama la atención por inesperado:

[...] el azar ha querido que mi reino esté constituido en su mayoría por mujeres, yo contentísima por supuesto con mi equipo ya que creo que poseemos todas las características necesarias para obtener el trono de hierro disfrutando en el camino y creando cosas sorprendentes.(+ 7$)$.

Nos incita a indagar sobre la pertenencia al grupo. Se siguen marcando las distancias por sexo, tendiendo a identificarnos y sentirnos más cómodos o cómodas con nuestros iguales, especialmente si de competir se trata. Esta arista de la gamificación, que habitualmente organiza los grupos al azar, ha sido poco trabajada, especialmente porque el campo emocional en el ámbito escolar no ha tenido protagonismo, siendo poco investigado (PAOLONI; VAJA; MUÑOZ, 2014). 
¿Por qué baja la satisfacción a medida que avanza la propuesta? Cuantitativamente es cierto. Bajan las narrativas en el diario común de reflexión, pero se mantiene vivo el sentimiento de máxima satisfacción con la propuesta. La calificación pasa a segundo plano y aparece de lleno el aprendizaje. Esta idea queda definida en esta declaración de una alumna cuando dice que no le "[...] importa la nota final, es que ya con todo lo vivido estoy muy satisfecha." (q 3).

El trabajo en equipo es otro de los motivos que provoca el sentimiento de satisfacción:

[...] tuve la suerte de caer con 8 personas, que si te soy sincero.... si me hubieran dado la opción de elegir a 8, tal vez no hubiera cogido a ninguna... Y hoy estoy encantado con cada uno de ellos... Porque los habrá más listos, más guapos y más ricos como diría un gran amigo mío, pero más implicados, comprometidos y voluntariosos seguro que no los hay. ( $\left.0^{\lambda} 22\right)$.

Se logra la cohesión a pesar de ser grupos seleccionados al azar. Sin azar, la tendencia es a crear los grupos de trabajo por afinidad, perdiéndose la oportunidad del descubrimiento del otro.

Les motiva ser situados en la incertidumbre y frente a un reto a lograr. Igual que el efecto de la subida de la temperatura provoca la licuación de las capas más profundas del permafrost siberiano; la provocación desde una metodología activa provoca en el alumnado que emerja su capacidad creativa y descubran lo: "... bonito [que] es aprender siendo feliz y disfrutando de lo que haces..." ( $q$ 14). Manifiestan que aprenden a escuchar y a dialogar: "Lo que más me gustó fue ver las diferentes perspectivas y conclusiones que cada persona saca

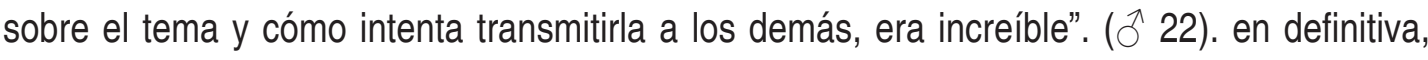
apostar por un aprendizaje dialógico.

\section{3 ¿HACÍA QUIENES SE DIRIGEN LAS EMOCIONES?}

Los sentimientos se disparan o se aplacan en función de los actores de la experiencia hacia quienes las dirigimos (grafo 3).

Grafo 3 - Presencia de Sentimientos y Emociones según los actores en el proceso

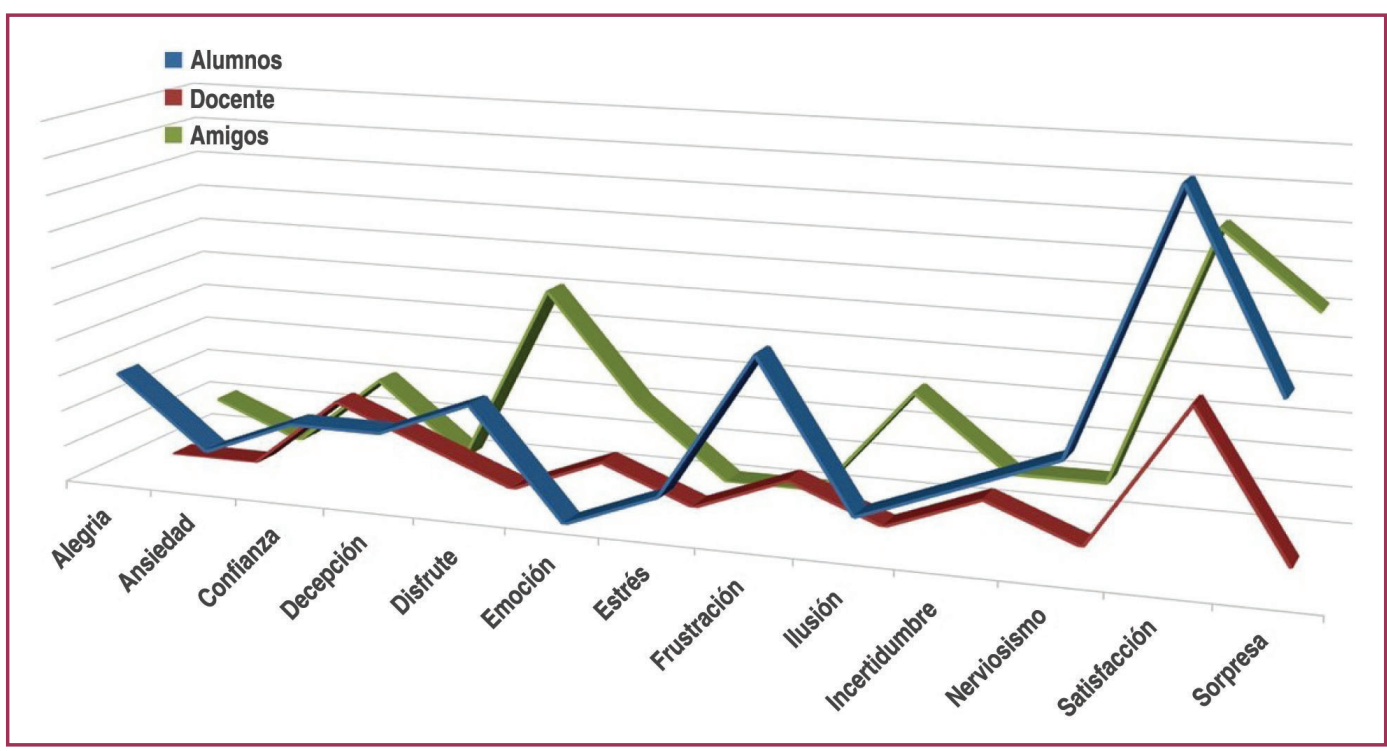

Fuente: elaboración propia 
Tenemos dos sentimientos constantes: la confianza y la satisfacción. La confianza transmitida por el docente la capta el alumnado "[...] nadie dijo que sería fácil y realmente acepté esto porque soy de los que cree en el trabajo y en las personas, y en el profesor se notaba confianza en nosotros, en ayudarnos al 100\%" ( $ぇ$ 22). Estamos de acuerdo con Herranz (2013) cuando plantea que el papel del docente debe ser activo, ha de ser el primer jugador y no debe situarse al margen de la experiencia.

La satisfacción se manifiesta en relación a los compañeros de equipo. Aparece la cohesión. El reconocimiento del grupo es lo importante, ya que no se sienten seres individuales, sino que entre todos forman un organismo vivo capaz de trabajar de forma cooperativa para lograr el objetivo. Disfrutar de la experiencia, eso es lo verdaderamente importante: "[...] Recordamos lo que llevábamos varios días hablando, estamos aquí para disfrutar, aprovechemos lo que estamos viviendo y olvidemos lo que nos jugamos, si caíamos volveríamos a intentarlo aún con más fuerza." (q 4).

Un docente que transmite confianza, ilusión e incertidumbre y, en algunos casos, ¿Por qué no?; frustración. El fracaso no es negativo; sirve para poner en acción la reflexión y autocrítica y diseñar un nuevo plan. En esta misma línea Foncubierta y Rodríguez (2014), nos alertan que el estudiante debe perder el miedo a fracasar, situación poco habitual en nuestras aulas, donde el predominio del éxito y la ocultación del fracaso prevalece.

\section{CONCLUSIONES}

Desde nuestra experiencia podemos decir que la gamificación, como estrategia metodológica en la Enseñanza Superior mejora la motivación del alumnado y aumenta su implicación. Dónde localizar los hallazgos de la afirmación. Básicamente en el sentimiento de satisfacción que queda marcado en el alumnado a lo largo de todo el proceso. En convergencia aparece el sentimiento de disfrutar con una propuesta novedosa, lejos del resto de materias, ofreciendo el punto inicial de motivación que todo aprendizaje requiere.

El clima de clase se basa en la confianza entre todos los actores: Cada propuesta supone un reto que provoca incertidumbre. El equipo se pone a buscar soluciones, sufriendo, frustración y decepción ante el error, pero sintiendo que tener que retroceder para volver a empezar ya lleva a aparejado el aprendizaje del proceso.

Debemos dar especial valor a la pertenencia al grupo. La forma de su creación pasa a un segundo plano; lo valioso es ser capaces de caminar como equipo, con el sentimiento de confianza puesto en el otro; con la capacidad de emocionarse por el éxito grupal y no el individual. Como diría "El árbol corazón": si no eres parte de la solución, es porque eres parte del problema. 


\section{REFERENCIAS}

BEZA, Olga. Gamification: How games can level up our everyday life? Ámsterdam: Universidad de Amsterdan, 2011. Disponible en: http://www.cs.vu.nl/ eliens/ct/local/material/gamification.pdf. Acceso el: 27 abr. 2019.

CAROLEI, Paula. Gameout: o uso de "gamification" para favorecer a imersão em diversos espaços pedagógicos in ensino superior. In: CONGRESSO INTERNACIONAL TIC E EDUCAÇÃO: HACIA LA EDUCACIÓN, 2012., 2. [Anales...]. Lisboa: Instituto de Educación de la Universidad de Lisboa, 2012.

CARPENA, Nicolás; CATALDI, Mariano; MUÑIZ, Gonzalo. En busca de nuevas metodologías y herramientas aplicables a la educación. Repensando nuestro rol docente en las aulas. In: CONGRESO IBEROAMERICANO DE GRÁFICA DIGITAL, 2102, 16., p. 633-6352012. Disponible en: http://papers.cumincad.org/data/works/att/sigradi2012 85.content.pdf. Acceso el: 12 abr. 2019..

CASASSUS, Juan. La educación del ser emocional. 2. ed. Santiago de Chile: Cuarto Propio 2007.

DAMASIO, Antonio. En busca de Spinoza: Neurobiología de la Emoción y los Sentimientos. Barcelona: Crítica, 2005.

DANOWSKA-FLORCZYK; Emilia; MOSTOWSKI, Piotr. Gamification as a new direction in teaching Polish as a foreign language. In: Edition of the International Conference "Ict For Language Learning", 5., 2012. Florencia: Pixel, 2012.

DENZIN, Norman K.; LINCOLN, Ivonna (Coords.). Manual de investigación cualitativa. Barcelona: Gedisa, 2012.

DETERDING, Sebastián; DIXON, Dan; KHALED Rilla; NACKE, Lennart. From Game Design Elements to Gamefulness: Defining "Gamification". In: Proceedings of the 15th International Academic MindTrek Conference: Envisioning Future Media Environments. Tampere: ACM, 2011. Disponible en: http://gamification-research.org/wp-content/uploads/2011/04/02-DeterdingKhaled-Nacke-Dixon.pdf. Acceso el: 20 abr. 2019.

ERENLI, Kai. The Impact of Gamification: a recommendation of Scenarios for Education. In: INTERNATIONAL CONFERENCE ON INTERACTIVE COLLABORATIVE LEARNING. 15. Villach: IEEE, 2012. Disponible en: https://ieeexplore.ieee.org/stamp/stamp. isp?tp=\&arnumber $=6402106$. Acceso el: 20 abr. 2019

ESTEBARANZ, Araceli. La enseñanza como tarea del profesor. In: MARCELO, Carlos. La función docente. Madrid: Síntesis, 2001. p. 103-140.

FONCUBIERTA, José Manuel; RODRIGUEZ, Chema. Didáctica de la gamificación en la clase de español. Madrid: Edinumen, 2014.

GAME MARKETING. Estudio 2012 Gamificación, expectativas y grado de adopción en España. Madrid: Edinumen, 2012.

GONZÁLEZ RODRÍGUEZ, Maria del Mar; DÍEZ LÓPEZ, Marta; LÓPEZ GAVIÑO, Francisca; ROMÁN RODRIGUEZ, Maite. La importancia del clima emocional del aula desde la perspectiva del alumnado universitario. Revista de Enseñanza Universitaria, n.35, p. 16-27, 2010.

Disponible en: http://institucional.us.es/revistas/universitaria/35/art 2.pdf. Acceso el: 18 ene. 2019 
GRIEVE, Ann MacDonald. Exploring the characteristics of 'teachers for excellence': teachers' own perceptions. European Journal of Teacher Education, v. 33, n.3, p. 265-277, 2010.

GROH, Fabian. Gamification: State of the Art Definition and Utilization. In: SEMINAR ON RESEARCH TRENDS IN MEDIA INFORMATICS, 4. [Proceedings...]. Ulm: Institute of Media Informatics University, 2012. p.39-46.

HERNÁNDEZ ÁLVAREZ, Juan Luis. La formación del profesorado de educación física: nuevos interrogantes, nuevos retos. Tándem, n.1, p. 53-66, 2000.

HERRANZ, Eduardo. Gamification: I Feria Informática. Madrid:Universidad Carlos III. 2013. Disponible en: http://revista.cbce.org.br/index.php/RBCE/article/viewFile/382/326. Acceso el: 25 mar. 2019

HUOTARI, Kay; HAMARI, Juho. Defining gamification-a service marketing perspective. In: INTERNATIONAL ACADEMIC MINDTREK, 16. [Proceedings...]. Tampere: ACM, 2012. Disponible en: http://doi.acm.org/10.1145/2393132.2393137. Acceso el: 27 may 2019.

KAPP, Karl. M. The Gamification of learning na instruction. San Francisco: John Wiley, 2012.

KUMAR, Balraj. Gamification in education-learn computer programming with fun. International Journal of Computers and Distributed Systems, v.2 , n.1, p. 46-53, 2012.

LEE, Joey J.; HAMMER, Jessica. Gamification in Education: What, How, Why Bother. Academic Exchange Waterly, v.15, n.2, 2011. Disponible en: https://www.uwstout.edu/soe/profdev/ resources/upload/Lee-Hammer-AEQ-2011.pdf . Acceso el: 9 abr. 2019.

MAURI, Teresa; COLL, César; ONRUBIA, Javier. La evaluación de la calidad de los procesos de innovación docente universitaria. Una perspectiva constructivista. Red U. Revista de Docencia Universitaria, v.1, 2007. Disponible en: http://red-u.net/redu/files/journals/1/articles/47/public/47-362-PB.pdf. Aceso el: 18 abr. 2019.

MCCOMBS, Bárbara L.; WHISLER, Jo Sue. La clase y la escuela centradas en el aprendiz. Barcelona: Paidós, 2000.

MELCHOR, Elías. Gamificación y e-learning. Un ejemplo con el juego del pasapalabra. In: EFQUEL Innovation Forum 2012. "Learning for Open Innovation. Transformation and Change for Future Learning". Granada: Universidad de Granada, 2012.

MÉNDEZ-LEITE, Analía E.; CORTÉS-GONZÁLEZ, Pablo; RIVAS-FLORES, José I. Narrativa y creatividad en la universidad. ¿es posible transitar otros caminos en la enseñanza y el aprendizaje? In : CORTÉS GONZÁLEZ, Pablo ; María Jesús; MÁRQUEZ GARCÍA (eds.), Creatividad, comunicación y educación: más allá de las fronteras del saber establecida. Málaga: Universidad de Málaga, 2017. p. 151-164.

MUNTEAN, Cristina. I. Raising engagement in e-learning through gamification. In: INTERNATIONAL CONFERENCE ON VIRTUAL LEARNING, 6. Bucharest : University of Bucharest, 2011. Disponible en: http://icvl.eu/2011/disc/icvl/documente/pdf/met/lCVL ModelsAndMethodologies paper42.pdf. Aceso el: 18 abr. 2019.

PAOLONI, Paola V.; VAJA, Arabela B; MUÑOZ, Verónica L. Reliability and Validity of the Achievement Emotions. Questionnaire. A Study of Argentinean University Students. Electronic Journal of Research in Educational Psychology, v.12, n.3, p. 671-692, 2014. 
PÉREZ-SAMANIEGO, Víctor; FUENTES Jorge M.; DEVÍS-DEVIS, José. El análisis narrativo en la educación física y el deporte. Movimento, v 17, n. 4, p. 11- 42, 2011.

PIANTA, Robert; BELSKY Jay; HOUT, Renate; MORRISON, Fred. Opportunities to learn in America's Elementary Classrooms. Science, n.315, p. 1795-1796, 2007. Disponible en: http:// citeseerx.ist.psu.edu/viewdoc/download?doi=10.1.1.624.7753\&rep=rep1\&type=pdf. Acceso el: 16 ene. 2019.

SIMÕES, Jorge; AGUIAR, Ademar; REDONDO, Rebeca; VILAS, Ana. Aplicação de elementos de jogos numa plataforma de aprendizagem social. In: CONGRESSO INTERNACIONAL TIC E EDUCAÇÃO: HACIA LA EDUCACIÓN, 2., 2012. Lisboa: Instituto de Educación de la Universidad de Lisboa. 2012. Disponible en: http://ticeduca.ie.ul.pt/atas/pdf/243.pdf. Acceso el: 27 ene. 2019.

SIMÕES, Jorge; DÍAZ, Rebeca; FERNÁNDEZ, Ana. A social gamification framework for a K-6 learning platform. Computers in Human Behavior, n.29, p. 345-353, 2013. Disponible en: https://www.sciencedirect.com/science/article/pii/S0747563212001574. Acceso el: 18 ene. 2019.

SPALEK, Klara; FASTENRATH, Matthias; ACKERMANN, Sandra; AUSCHRA, Bianca; COYNEL, David; FREY, Julia; GSCHWIND, Leo; HARTMANN, Francina; VAN DER Nadine A.; PAPASSOTIROPOULOS, Maarel; DE QUERVAIN, Dominique; MILNIK, Annette. SexDependent Dissociation between Emotional Appraisal and Memory: A Large-Scale, Behavioral and fMRI Study. Journal of Neuroscience, 2015. Disponible en: http://www.jneurosci.org/ content/35/3/920.full. Acceso el: 26 mar. 2019.

WERBACH, Kevin; HUNTER, Dan. For the Win: How Game Thinking Can Revolutionize Your Business. Pennsylvania: Wharton Digital, 2012. 